Apidologie, 1980, 11 (1), 17-24.

\title{
ZUR HALTUNG VON APIS MELLIFICA IN EINEM FLUGRAUM
}

\author{
Ulrike ROTHE und Werner NACHTIGALL* \\ Zoologisches Institut der Universität des Saarlandes \\ Fachbereich 16 \\ D - 66 Saarbrücken Fachr. 4 (B.R.D.)
}

\begin{abstract}
SUMMARY
THE KEEPING OF APIS MELLIFICA IN A FLIGHT ROOM (HYMENOPTERA APIDAE)
\end{abstract}

The conception and construction of a non-expensive air-conditioned flight room for honeybees are described. First experiences with a hive containing 7 combs of Apis mellifica carnica are given.

\section{ZUSAMMENFASSUNG}

Es werden Konzeption und Bau eines kostengünstigen, klimatisierten Flugraumes (FR) für Bienen beschrieben sowie erste Erfahrungen mit einem 7 Waben Volk der Carnicabiene (Apis mellifica carnica) mitgeteilt, das seit Dezember 1978 in diesem FR gehalten wird.

\section{1. - EINLEITUNG}

Zum Zwecke der ganzjährigen Verfügbarkeit von flugfähigen Arbeitsbienen für insektenphysiologische Untersuchungen sind Bienenstände im Freiland nicht ausreichend. In solchen Fällen bietet sich die Haltung von mindestens einem Bienenvolk in einem klimatisierten FR an. Unsere Anforderungen an einen solchen FR richteten sich nach den gegebenen Räumlichkeiten und berücksichtigten die Erfahrungen mit modernen Flugräumen in Deutschland. Die von Velthuis-van

\footnotetext{
* Mit freundlicher Unterstützung der Deutschen Forschungsgemeinschaft.
} 
PraAGH $(1972,1974)$ vorgeschlagene Bauweise - ein ringsum mit Gardinennetz bespanntes Dexionstabgestell in einem vollklimatisierten Raum - war bei uns aus Platzgründen nicht möglich. Im Kehlbalkenspeicher des Institusgebäudes wurde deshalb unter Ausnutzung der vorhandenen Stützbalken ein FR ohne umgebende Klimakammer gebaut, der in Anlehnung an das Rennersche (RENNER, 1955) Flugraumkonzept viele technische Einzelheiten eines modernen FR wie bei van Praagh (1976), Ruttrer (1977), Lindauer und Martin (1977) und Douault (1978) auf sehr preisgünstige Weise verwirklicht und Anregung für Nachbauten geben kann. Im folgenden sind alle wesentlichen konstruktiven Merkmale dieses neuen FR erläutert (s. Abb. 1).

\section{II. - TECHNISCHE ANGABEN ZUM FR}

Die Grundfläche des FR beträgt 2,05 $\mathrm{m} \times 4,13 \mathrm{~m}$ bei $2,30 \mathrm{~m}$ Höhe. Die Wände sind unter Verwendung von Blechprofilen in Sandwich-Bauweise erstellt. Am Blechprofil sind aussen $12 \mathrm{~mm}$ starke Rigipsplatten und innen $16 \mathrm{~mm}$ starke Presspanplatten mit weisser Kunststoffbeschichtung angebracht. Zur Isolierung befindet sich zwischen Aussen- und Innenwand eine $5 \mathrm{~cm}$ starke Schicht von Styropor. Als Beleuchtungskörper dienen 36 Neonröhren des Typs Osram L, 40 W/25 Universal Weiss/White von $1,20 \mathrm{~m}$ bzw. $1,50 \mathrm{~m}$ Länge, die in 6 doppelten Längsreihen zu je $2 \times 3$ Stück an der Decke angeordnet sind. (Auf den Lampentyp Philips TL 05 wurde unter anderem aus Gründen des gesundheitlichen Schutzes des FR-Benutzers vor Schädigungen durch den hohen UV-Anteil dieses Röhrentyps verzichtet.) Um in einer Ebene annähernd symmetrische Beleuchtungsverhältnisse mit geeigneter Flickerfrequenz zu erzielen, sind die Neonröhren abwechselnd an jeweils eine Phase von Dreiphasenwechselstrom angeschlossen, so dass sich eine Flimmerfrequenz von $300 \mathrm{~Hz}$ ergibt. Zur Kühlung der Deckenbeleuchtung sind an den Längswänden in der Höhe der Röhren Ventilatoren angebracht. Die Beleuchtung kann wahlweise im Handbetrieb oder über eine Zeitschaltuhr gestellt werden. Ausser diesen Röhrenpaaren ist noch eine Einzelröhre als Arbeitsbeleuchtung und ein kleines Pilotlicht $(6 \mathrm{~V}, 2$, 4 Watt) zur nächtlichen Beleuchtung am Stockeingang installiert. Zur Luftbefeuchtung ist eine Wasserwanne mit Verdunstersaugeinlagen aufgestellt; mit dieser einfachen Einrichtung werden ausreichende Raumfeuchten von maximal $53 \%$ (rel. Feuchte) erreicht. Zur Erwärmung dient eine Warmwasserfussbodenheizung mit verstellbarem Thermostaten. Die Heizung ist so ausgelegt, dass bei $-15^{\circ} \mathrm{C}$ Aussentemperatur eine Raumtemperatur von $28^{\circ} \mathrm{C}$ einstellbar ist. Für diese Temperaturen berechnet sich der Gesamtwärmebedarf im FR zu ca. $1200 \mathrm{Watt}$, das entspricht rund $130 \mathrm{Watt} / \mathrm{m}^{2}$ bei einer Fläche von $9 \mathrm{~m}^{2}$. Als Heizrohre sind 54 laufende Meter Polyäthylen-Rohre mäanderartig in vorgefertigten Schamottsteinen ausgelegt. Zur Isolierung nach unten dient Styropor, zum FR hin ist mit verzinktem Stahlblech abgedeckt. Für die Frischluftversorgung wurde ein richtungs- und drehzahlverstellbarer Axialventilator montiert. Frische, kühle Luft kann aus dem darunterliegenden Geschoss angesaugt 


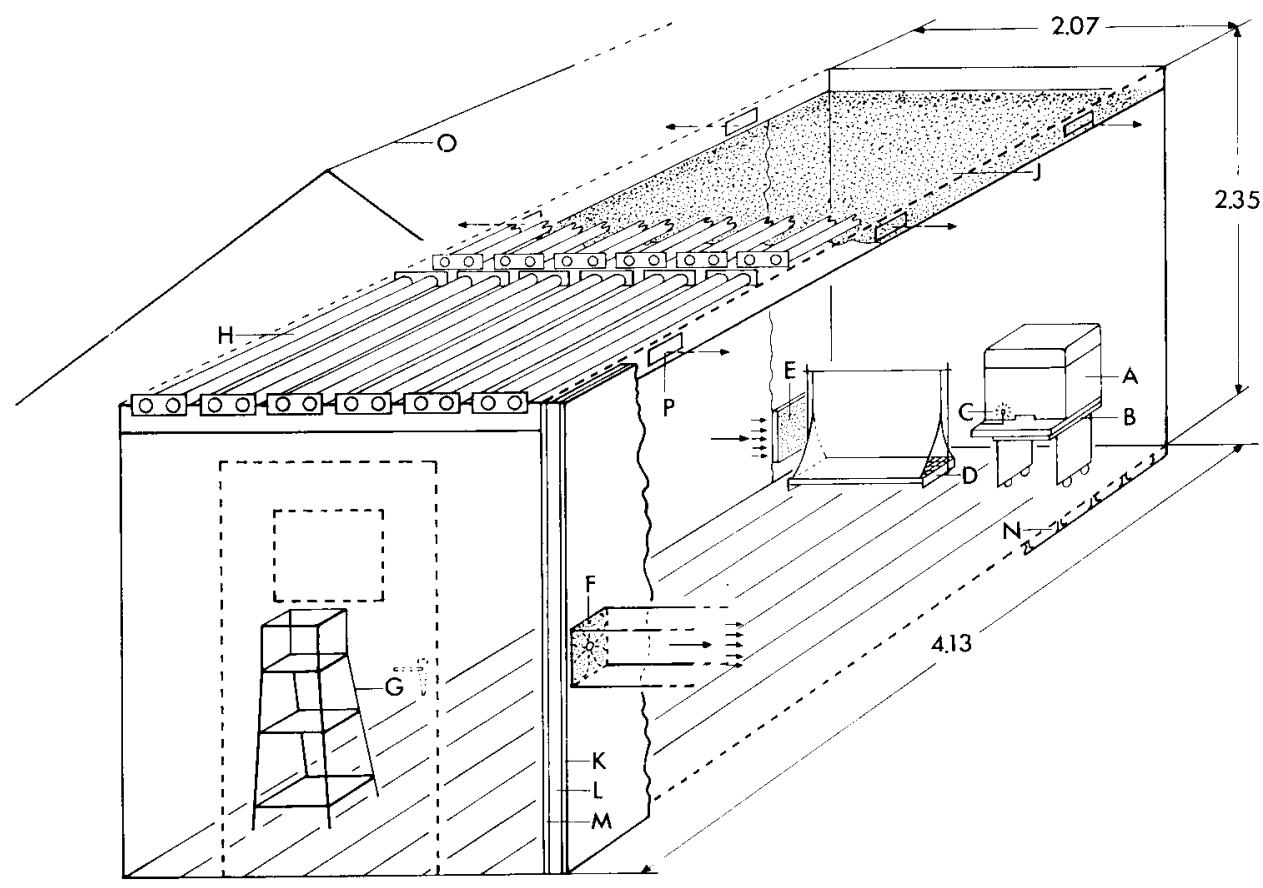

Авв. I. - Bienenflugraum

$\mathrm{A}=$ Beute; $\mathrm{B}=$ Schaumgummiunterlage $; \mathrm{C}=$ Fluglochbeleuchtung; $\mathrm{D}=$ Befeuchter $; \mathrm{E}==$ Frischluftkanal; $\mathrm{F}=$ Ventilator $\mathbf{G}=$ Futterhocker $; \mathrm{H}=$ Deckenbeleuchtung; $\mathrm{J}=$ Gardine; $\mathrm{K}=$ Rigipsplatte $\mathrm{L}=$ Styropor; $\mathrm{M}=$ Pressspanplatte; $\mathrm{N}=$ Fussbodenheizung; $\mathrm{O}=$ First $\mathrm{P}=$ Lüftungsschlitze. Alle Massangaben in $\mathrm{m}$.

FIG. I. - Chambre de vol.

$\mathrm{A}=$ ruche $\mathbf{B}=$ socle en caoutchouc mousse; $\mathrm{C}=$ éclairage du trou de vol; $\mathrm{D}=$ humidificateur $\mathrm{E}=$ canal d'aération; $F=$ ventilateur; $G=$ escabeau pour le nourrissement; $H=$ éclairage du plafond; $J=$ rideau; $\mathrm{K}=$ carreaux de plâtre; $\mathrm{L}=$ isolant (polystyrène expansé); $\mathrm{M}=$ planche d'aggloméré; $\mathrm{N}=$ chauffage par le sol; $\mathrm{O}=$ arête du toit; $\mathrm{P}=$ ouverture de ventilation. Les mesures sont en mètres.

werden. Alle Wandöffnungen und die Deckenbeleuchtung sind mit leicht auswechselbaren Gardinennetzen gegen die Bienen abgegrenzt. Fussbodenbelag und Wände werden aus hygienischen Gründen mit Zeitungspapier abgedeckt bzw. beklebt.

\section{III. - HALTUNGSBEDINGUNGEN}

Zur Zeit wird im FR ein Bienenvolk der Rasse Apis mellifica carnica gehalten. Die Beute steht aus praktischen Gründen auf einem fahrbaren Untersatz und zur Vibrationsdämpfung auf einer Schaumgummiunterlage. Die Luftfeuchte im FR betrug ca. $20-50 \%$ rel. Feuchte, abhängig vom Messpunkt, der Raumtemperatur und dem 
Alter des Zeitungsbelages. Die Raumtemperatur schwankte im Beobachtungszeitraum am Tage und in der Nacht sowie am Boden und an der Decke um einen Wert von $23 \pm 5^{\circ} \mathrm{C}$ ( \pm grösste absolute Abweichung). Der Luftdruck schwankte mit dem Aussendruck. Die Beleuchtungsstärke betrug zwischen 2000 und 4000 Lux. Im FR herrschte ein Tag/Nacht-Rhythmus von 11/13 Stunden ohne zusätzliche jahreszeitliche Periodik. Alles Futter wird auf einem mehretagigen "Futterhocker " angeboten, der durch ein Beobachtungsfenster eingesehen werden kann. Tiefgefroren gemahlener Pollen wird auf einer Gesamtfläche von ca. $50 \mathrm{~cm}^{2}$ ad libitum angeboten. Ausserdem wird handelsüblicher Rohrzucker $45 \%$ ig, 0,1\% ig an Fumidil B, gereicht. Wasser kann am Futterplatz und an der Befeuchteranlage gesammelt werden. Im Stock werden ausserdem wöchentlich 1-2 leere Randwaben mit Leitungswasser aufgefüllt.

\section{IV. - INBETRIEBNAHME DES FR}

Am 5-12-1978 wurde ein 7-Waben Volk der Rasse Apis mellifica carnica in den FR gestellt. Innerhalb der ersten 5 Stunden nahm die Flugaktivität sprunghaft zu. Am erstem Tag hatte keine Biene Futterkontakt. Die dann mit Anisöl aromatisierte Futterstelle wurde am 2. Tag akzeptiert. Die Pollensammelaktivität stieg bis auf einen Wert von 40-50 Sammlerinnen/Gesamtfläche gleichzeitig an. Zur Eingewöhnung wurde zunächst 2 Tage lang Dauerlicht geboten.

14 Tage nach Inbetriebnahme wurde der Zustand des Volkes kontrolliert. Die Wintertraube war aufgelöst, 2 Waben hatten einige Stifte, eine davon enthielt schon auf wenigen Quadratzentimetern unverdeckelte Brut. Nach weiteren 14 Tagen waren deutlich grössere Pollenvorräte sichtbar, das Brutnest hatte sich auf 3 Waben ausgedehnt und auf jeder Wabe vergrössert. Nach 2-monatiger Eingewöhnungszeit wurde am gesunden Volk mit der Altersbestimmung der Arbeiterinnen begonnen.

\section{V. - ALTERSBESTIMMUNG}

Es wurden 10 Tage lang 20 eben schlüpfende Jungbienen numeriert und von oben in den Stock zurückgesetzt. Das Markieren dauerte täglich maximal 1/2 Stunde. Der tägliche Totenfall wurde nach markierten Bienen durchgesehen; zur Kontrolle wurde regelmässig im Stock nach numerierten Bienen gesucht. Die bei dieser Lebensdaueranalyse erzielten Daten können maximal \pm 1 Tag vom tatsächlichen Wert abweichen, das entspricht bei dem, vorliegenden Ergebnis einem absoluten Fehler von kleiner als $2.5 \%$, bezogen auf das mittlere Lebesalter. Zur Qualitätskontrolle des Tiermaterials wurde während des Testzeitraumes eine Merkmalsuntersuchung an 100 Einzeltieren nach dem Vorschlag des Deutschen Imkerbundes durchgeführt. Neben dem Cubitalindex waren Haarlänge, Panzerzeichen und Filzbinden Parameter. Im Testzeitraum wurde ausserdem die Sterberate protokolliert. 


\section{VI. - ERGEBNISSE UND DISKUSSION}

Die Ergebnisse der Altersbestimmung und Merkmalsuntersuchung sind in Abbildung 2 dargestellt. Die erhaltene Merkmalsformel (s. Abb. 2 (2)) und die Verteilung der' Cubitalindices sprechen für ein homogenes Ausgangsmaterial. Die Bestimmung des Lebensalters ergab einen Mittelwert von $45 \pm 16$ Tagen (Standardabweichung) minimale und maximale Werte lagen bei 24 bzw. 92 Tagen. Weil die Literaturangaben über das Lebensalter von Freilandbienen während der Frühlings - und Sommermonate sehr heterogen sind, ist das Ergebnis schwierig einzuordnen. Am häufigsten wird jedoch ein Alter von 4-5 bzw. 5-6 Wochen angegeben [Frisch (1969), Grout, Ruttner (1973), Peschetz (1954), Butler (1954), HornsmanN (1956), Leuenberger (1948), RAutenberg (1946), RibBands (1953)]. Flugraumbienen sollen länger als Sommerbienen im Freiland leben IMARTIN (1977), KELLNER und JACOBS (1977)]. Die vorliegende Untersuchung scheint dies zu bestätigen, zur Sicherung ist eine mehrfache Wiederholung vorgesehen.

Die Messung des täglichen Totenfalls ergab den in Abbildung 2 dargestellten Verlauf. Unregelmässigkeiten in der Sterberate sind - gleiche durchschnittliche Lebensdauer der Einzeltiere vorausgesetzt - mit einer diskontinuierlichen Legeaktivität der Königin zu erklären. Dies wird durch die Beobachtung gestützt, dass während des Testzeitraumes gelegentlich nur eine sehr geringe Anzahl von Stiften zu finden war. Dieses Legeverhalten kann verschiedene Ursachen haben. Es ist vom Gesundheitszustand des Volkes und stockexternen FR-Bedingungen wie Feuchte, Temperatur etc. abhängig. Die Feuchtigkeit der Stockluft kann wegen der beschriebenen regelmässigen Wassergabe im Stock [LINDAUER (1959)] kein hemmender Faktor für die Brutaktivität sein; sie liegt oberhalb des von van PRAAGH (1975) angegebenen Mindestwertes von 19,5 mbar (entsprechend 36,6\% rel. Feuchte bei $34{ }^{\circ} \mathrm{C}$ ) zur Aufrechterhaltung des Brutgeschäftes. (Bei einer mittleren Brutnesttemperatur von $34,3 \pm 0,2^{\circ} \mathrm{C}$ (maximale absolute Abweichung) ergab sich ein Mittelwert von der relativen Feuchte von $58 \pm 7 \%$ entsprechend $31,1 \pm 3,7 \mathrm{mbar}$ ). In diesem Zusammenhang wird auch die Abhängigkeit der Sammelaktivität von der Fläche des künstlichen Futterplatzes diskutiert [VESELY (1977)]. Eine Vergrösserung der Pollensammelfläche in unserem FR erscheint nicht notwending, da grosse Pollenvorräte in den Stock eingetragen wurden. Die Futterstelle scheint ausserdem hinreichend attraktiv zu sein, was sich in beobachteten Tänzen von Pollensammlerinnen auf den Waben widerspiegelt. Die allgemeine Stagnation des Brutgeschäftes bei relativ geringer Brutwabenzahl wird von FR-Völkern immer wieder berichtet [Czoppelt (1977), Velthuis (1977)] und unter anderem im Zusammenhang mit Überbevölkerung diskutiert. Dieser hemmende Faktor ist bei unseren FR-Massen nicht auszuschliessen, kann aber nicht der allein massgebliche sein, da ähnliche Schwierigkeiten auch in weitaus grossräumigeren Flugräumen die Regel sind.

Insgesamt ist die bisherige einhalbjährige Erfahrung mit dem geschilderten FR durchaus gut. Der zeitliche Aufwand für die Pflege des Raumes ist relativ gering, er 

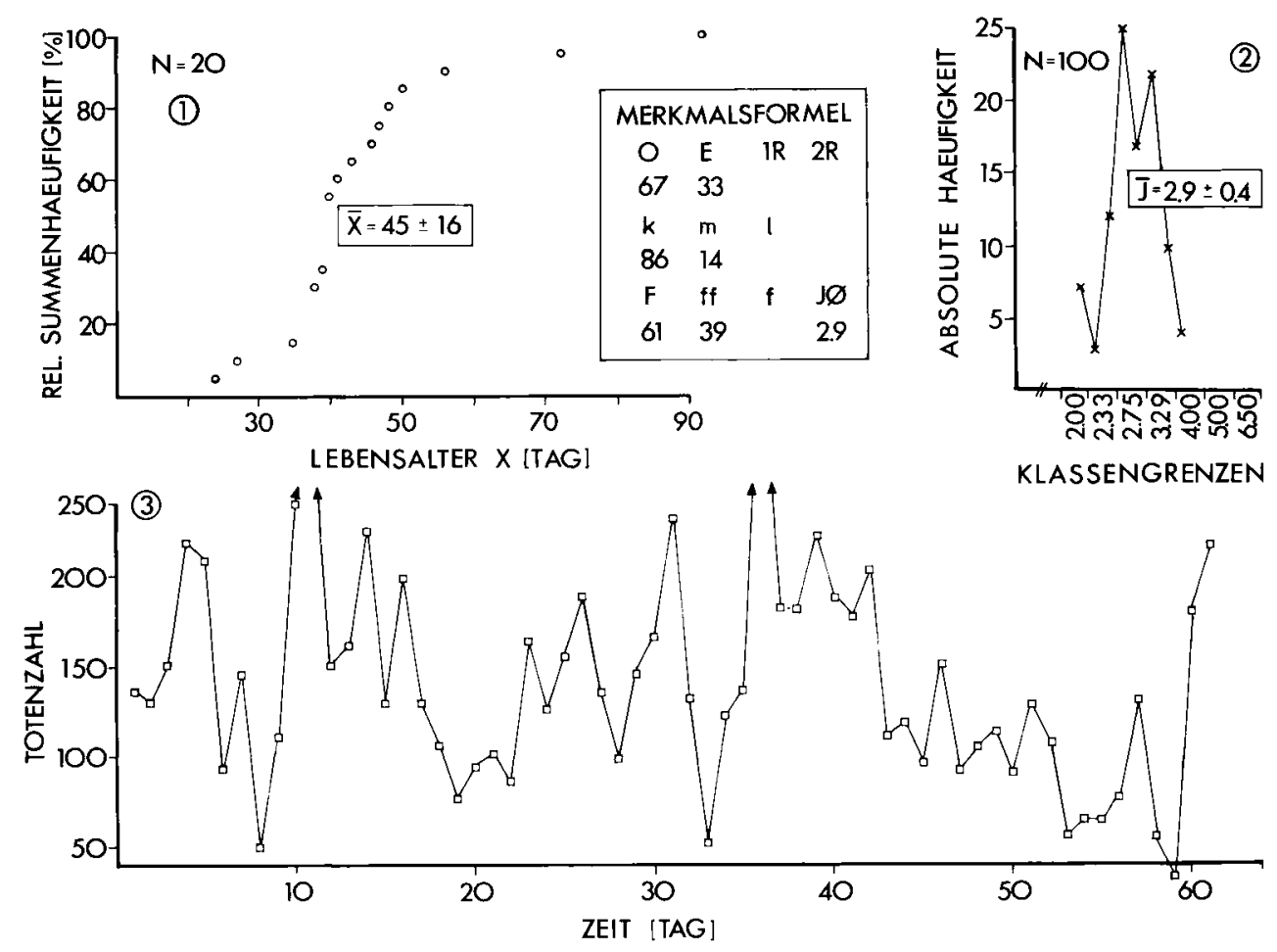

Aвв. 2. - Ergebnisse der Altersbestimmung (1), der Untersuchung der Rassenmerkmale (2), sowie zeitlicher Verlauf der Sterberate (3).

Erläuterungen zu den Abkürzungen der Merkmalsformel :

- Panzerzeichen (Farbzeichen an der breitesten Hinterleibsschuppe) : $\mathrm{O}=$ ganz schwarz, ohne deutliche gelbe Zeichen; $\mathrm{E}=$ deutlich gelbe Farbzeichen ohne Rückenverbindung; I $\mathrm{R}=1$ gelber Ring; $2 \mathrm{R}=2$ gelbe Ringe.

- Haarlänge (Sattelhaarlänge auf dem Tergit des 5. Abdominalsegmentes) : $\mathrm{K}=$ unter $0,35 \mathrm{~mm} ; \mathrm{m}=$ $0,35-0,4 \mathrm{~mm} ; 1=$ über $0.4 \mathrm{~mm}$.

- Filzbinden (Breite der mittleren Binde 2 seitlich ihrer breitesten Stelle im Vergleich zur Breite des schwarzen Segmentteils) $: F=$ Binde deutlich breiter als schwarzer Rand; $f f=$ Binde und Rand ungefähr gleichbreit; $f=$ Binde schmaler als schwarzer Rand.

- $\mathbf{J}=$ Cubitalindex nach GoETzE (Verhältnis der beiden Grundadern der 3. Cubitalzelle).

FIG. 2. - Détermination de l'áge (1), étude des caractères raciaux (2), évolution dans le temps de la mortalité (3)

(1) en abcisses : durée de vie en jours; en ordonnées: fréquence cumulative relative.

(2) en abcisses : limite des classes; en ordonnées : fréquence absolue.

Abréviations utilisées dans la formule biométrique :

- Coloration du tergite le plus large : $\mathrm{O}=$ totalement noir, aucun signe jaune manifeste: $\mathrm{E}=$ signes de coloration jaune manifeste sans liaison dorsale; $1 \mathrm{R}=$ un anneau jaune: $2 \mathrm{R}=-2$ anneaux jaunes.

- Pilosité (longueur des poils du tergite du $5^{\mathrm{E}}$ segment abdominal) $: \mathrm{k}=>0,35 \mathrm{~mm} ; \mathrm{m}=-0,35-4,0 \mathrm{~mm}$; $1==0,4 \mathrm{~mm}$.

- Tomentum (largeur de la bande pileuse moyenne à son maximum en comparaison avec la largeur de la partie noire du segment) $: F=$ bande nettement plus large que la bordure noire; $f f=$ bande pileuse et bordure noire de même largeur environ; $\mathrm{f}=$ bande pileuse plus étroite que la bordure noire.

- J : index cubital d'après GoETze (rapport des 2 nervures de la $3^{e}$ cellule cubitale).

(3) en abcisses : temps (en jours); en ordonnées : nombre d'abeilles mortes. 
beträgt täglich etwa eine halbe Stunde. Der Gesundheitszustand des Volkes ist nach einem unbedeutenden Kalkbrutbefall im ersten Monat gut. Das mittlere Lebensalter scheint mit $45 \pm 16$ Tagen bei den erwarteten Werten zu liegen. Das Flugverhalten der FR-Bienen führt zu keiner mechanischen Verletzung der Flügel und die Futtersammelaktivität ist für die Aufrechterhaltung des Brutgeschäftes ausreichend.

\section{DANK}

Der technischen Abteilung der Universität des Saarlandes danken wir für das verständnisvolle Berücksichtigen von Sonderwünschen beim Bau des FR; Herr Dipl.-Biol. R. PAUL hat uns als erfahrener Imker mit vielen nützlichen Ratschlägen geholfen.

Eingegangen im August 1979

Reçu pour publication en août 1979

\section{RÉSUME}

\section{LE MAINTIEN D'APIS MELLIFICA DANS UNE CHAMBRE DE VOL}

On décrit la conception et la construction d'une chambre de vol pour abeilles, peu coûteuse et climatisée, dans laquelle une colonie d'Apis mellifica carnica est maintenue depuis décembre 1978. Pour des raisons de place il n'a pas été possible de réaliser celle du type Velthuis-van PraAGH - cage faite avec des supports en dexion recouverts d'un voilage dans une pièce entièrement climatisée. La chambre de vol a été édifiée en mettant à profit les poutres porteuses dans le grenier du bâtiment de l'Institut. La figure 1 montre les principaies caractéristiques de la construction. L'éclairage du plafond est constitué par 36 tubes au néon, qui sont reliés alternativement à chacune des phases du courant triphasé, de sorte qu'il en résulte une fréquence de scintillement de $300 \mathrm{~Hz}$. Les tubes au néon peuvent être refroidis par de petits ventilateurs situés dans les ouvertures de ventilation. L'éclairage du plafond est réglé par une horloge à contacts selon un rythme jour-nuit de 11-13 heures sans périodicité saisonnière supplémentaire. L'intensité lumineuse est comprise entre 2000 et 4000 lux. La nuit on allume l'éclairage du trou de vol. Pour le chauffage on a encastré un chauffage à eau chaude par le sol réglé par un thermostat. Pour le renouvellement de l'air un ventilateur axial aspire l'air frais par un canal d'aération venant de l'étage inférieur. La température de la chambre a oscillé au cours de la période d'observation (décembre 1978 à mai 1979) autour de $23 \pm 5{ }^{\circ} \mathrm{C}$ (écart absolu). Une cuve d'eau comportant un dispositif d'absorption-évaporation a été installée pour humidifier l'air. L'humidité de l'air dans la chambre de vol a atteint $20-50 \%$ d'humidité relative.

Comme nourrissent on a fourni un sirop de sucre de canne du commerce à $45 \%$ enrichi de $0,1 \%$ de Fumidil B. On a offert ad libitum du pollen congelé et broyé déposé sur une surface d'environ $50 \mathrm{~cm}^{2}$. Pour déterminer l'âge on a numéroté chaque jour pendant 10 jours 20 jeunes abeilles qui venaient d'éclore. On a examiné la mortalité journalière d'après les abeilles marquées et à titre de témoin elle a été relevée régulièrement dans la ruche d'après les abeilles numérotées. L'analyse de la durée de vie a donné une valeur moyenne de $45 \pm 16$ jours (écart standard) (voir fig. 2 (1). D'après ce premier résultat il semble que les abeilles de la chambre de vol vivent plus longtemps que les abeilles d'été en plein air. L'étude des caractéristiques biométriques faite sur 100 abeilles pour contrôler la qualité du matériel vivant a fourni la formule biométrique représentée dans la figure 2 (2). Cette formule et la répartition de l'index cubital témoignent d'un matériel homogène. Les résultats de la mortalité journalière, représentés sur la figure 2 (3), sont discutés en relation avec l'activité de ponte discontinue de la reine. Dans l'ensemble les résultats obtenus jusqu'à présent dans la chambre de vol avec la colonie carnolienne sont satisfaisants. 


\section{LITERATUR}

Butler C. G., 1954. - The world of the honeybee, Collins, St. James Place, London.

Czoppelt Ch., 1977. III. Verhalten von Bienenvölkern in einem Winterflugraum, Insectes sociaux, 24 (3), 261-300.

DouAult Ph., 1978. - L'évage expérimental des abeilles en milieu clos. La nouvelle salle d'élevage de la Station de Recherches de Bures-sur-Yvette, Bull. tech. apic. OPIDA, 5 (4), 15-22.

Fisch K. von, 1969. - Aus dem Leben der Bienen, Springer Verlag, Berlin, Heidelberg, N.Y.

Grout R. A. und Ruttner F., 1973. - Beute und Biene, Ehrenwirth Verlag, München.

Hornsmann E., 1956. - Die Welt der Biene, Kindler Verlag, München.

Kellner N. und JACOBS F. J., 1977. - VI. Möglichkeiten zur Verbesserung des Flugraumes nach van PRAAGH (1972), Insectes sociaux 24 (3), 261-300.

Leuenbergen F., 1948. - Der Schweizerische Bienenvater, Lehrbuch der Bienenzucht.

Lindauer M., 1954. - Temperaturregulierung und Wasserhaushalt im Bienenstaat. Z. iergl. Physiol. 36, $391-432$.

Martin H., 1977. - Pers. Mitteilung.

Peschetz H., 1954. - Vom Anfänger zum Meister, Carnica-Bienenbuch, Verlag Absatia, Colmar-Freiburg.

RAUtenberg H.J., 1956. - Moderne erfolgreiche Bienenzucht, Verlag der Deutschen Bienenzeitung.

RENNER M., 1955. - Über die Haltung von Bienen in geschlossenen künstlichen Räumen Naturwiss., 42, 539-540.

RIBBANDS R., 1953. - The behaviour and social life of the honeybee, Bee Research Association Limited, London.

RutTner F., 1977. - I. Entwicklung und gegenwärtiger Stand der Flugraumtechnologie, Insectes sociaux, 24 (3), 279-285.

Van PraAgh J. P., 1972. - Towards a controlled - environment room suitable for normal colony life of honeybees, J. apic. Res., 11 (2), 77-87.

VAN PraAgh J.P., 1974. - Doktorarbeit, De ontwilkeling von een klimaatkamer voor de honingbij, Utrecht.

VaAn Praagh J. P., 1975. - Die Feuchtigkeit der Stockluft und die Bruttätigkeit der Bienen (Apis mellifica L.) in einem Flugraum. Apidologie, 6 (3), 283-293.

VAAN PRAAGH, 1976. - Light ripple and visual acuity in a climate room for honeybees (Apis mellifica L.), Neth. J. Zool., 25 (4), 506-515.

Velthuis H.H.W., 1977. - V. Population dynamic aspect and communication in a bee flight room, Insectes sociaux, 24 (3), 293-296.

VesEly V., 1977. - VII. Stimulierung der Eiablage instrumentell besamter Königinnen im Flugraum, Insectes Sociaux, 24 (3), 297-299. 